\title{
Doble presencia: un riesgo psicosocial que evidencia la desigualdad entre hombres y mujeres en la conciliación de la vida familiar y laboral
}

\author{
Lucia Estevan Reina, Rosa $\mathrm{M}^{\mathrm{a}}$ Rodríguez Sánchez, Borja Romero González, Álvaro \\ Rodríguez López, Marina Romo Sola. Universidad de Granada
}

\author{
Supervisado por el profesor José Miguel García Ramírez. Departamento de Psicología Social. Universidad de Granada \\ Fecha de recepción: 30 de mayo de 2014. \\ Fecha de revisión: 5 de junio de 2014. \\ Fecha de aceptación: 5 de junio de 2014
}

\section{RESUMEN}

Los riesgos psicosociales son tema de actualidad en el mundo que nos rodea debido a los constantes cambios económicos y sociales. Más aún, la doble presencia, esto es, el trabajo realizado tanto como empleados/as remunerados/as, como el realizado en las tareas familiares y domésticas, generalmente de cuidados (Ramos, 2008). Este riesgo psicosocial afecta en mayor medida a mujeres que a hombres, ya que éstas poseen el rol de cuidadoras y protectoras del hogar. En el presente estudio se ha pretendido investigar este fenómeno en una muestra de 105 trabajadores de la UGR mediante el cuestionario ISTAS 21. Se comprobó a través de los análisis estadísticos pertinentes que existe una diferencia significativa que indica una mayor presencia de este riesgo psicosocial en mujeres que en hombres.

Palabras clave: Riesgos psicosociales, doble presencia, salud laboral.

\section{INTRODUCCIÓN}

Según la OMS entendemos por salud el estado de bienestar físico, psicológico y social y no solo la ausencia de enfermedad. El trabajo es algo indispensable para la vida para la mayoría de la población. Así pues no tener trabajo puede convertirse en un gran estresor, del mismo modo que puede serlo tener un trabajo con unas malas condiciones laborales.

Los riesgos psicosociales son aquellas condiciones que se encuentran presentes en el contexto laboral (entorno físico, ambiente social, contenido del trabajo, organización) y que tienen capacidad para afectar tanto al bienestar o la salud (física, psíquica o social) del trabajador como al desarrollo del trabajo (Instituto Nacional de Seguridad e Higiene en el Trabajo; Ley de Prevención de Riesgos Laborales (LPRL 21/1995, art. 4.7).

Estos riesgos pueden provocar efectos negativos sobre la salud derivados del trabajo (ya sean físicos o psicológicos) y enfermedades, patologías, lesiones, etc. causadas por el trabajo. Por lo cual se hace indispensable abordar esto a través de la promoción de la salud en los entornos de trabajo, que no sólo significa marcarse el objetivo de reducir las tasas de accidentabilidad y de enfermedades profesionales. Sino que debe orientarse a: la promoción y el mantenimiento del bienestar físico, mental y social de los/as trabajadores/as; la prevención de las alteraciones de salud; la protección del 
riesgo; el ambiente adaptado a las capacidades de los/as trabajadores/as; del trabajo al trabajador/a y viceversa.

Diferentes autores/as han propuesto diferentes clasificaciones de lo que podríamos considerar como riesgos psicosociales, no obstante el modelo en el que nos vamos a centrar agrupa dichos riesgos en seis dimensiones (Moncada, Llorens, Navarro y Krinstensen, 2005): exigencias psicológicas, trabajo activo y posibilidad de desarrollo, inseguridad, apoyo social y calidad de liderazgo, doble presencia y estima (dimensiones recogidas en el ISTAS21).

No obstante es preciso destacar también como el contexto de crisis socioeconómica en el que nos encontramos supone en sí mismo un estresor que puede agudizar algunos de los riesgos psicosociales mencionados, especialmente por la precarización de muchos puestos de trabajo (reducciones salariales, incremento de la jornada laboral, reducción de las medidas de seguridad en el empleo, descuelgues de los convenios colectivos, facilitación de los despidos...).

Por otro lado, cabe mencionar que algunas características de la población pueden también hacerlas más proclives a sufrir estos riesgos psicosociales en el ámbito laboral (edad, sexo, origen étnico..,), y precisamente en este aspecto es el que queremos centrarnos. Si bien, como hemos mencionado, los riesgos psicosociales asociados al contexto laboral son muchos, estamos interesados/as en abordar aquellos vinculados a la Doble Presencia. Se entiende como tal al trabajo realizado tanto como empleados/as remunerados/as, como al realizado en las tareas familiares, domésticas, generalmente de cuidados (Ramos, 2008); o como el hecho de que recaigan sobre una misma persona la necesidad de responder a las demandas del espacio de trabajo doméstico-familiar y a las demandas del trabajo asalariado, sería un riesgo para la salud que se origina por el aumento de la carga de trabajo así como por la dificultad para responder a ambas demandas cuando se producen de manera simultánea (Instituto Sindical de Trabajo, Ambiente y Salud, 2012).

El hecho de que la Doble Presencia sea un riesgo psicosocial habitual en las mujeres se debe a que tradicionalmente han sido quienes han organizado y realizado la mayor parte del trabajo doméstico familiar. Algunos datos que ponen de manifiesto este fenómeno son: el hecho de que mientras que las mujeres dedican de 5-6 horas diarias de media al trabajo doméstico, los hombres sólo dedican 2; que, en términos generales, incluso cuando la familia se mantiene únicamente a través del sueldo de la mujer es ella quien sigue realizando las tareas domésticas en mayor medida, que el estado de salud de las mujeres es peor cuanto mayor sea el número de personas en el núcleo familiar. (Instituto Sindical de Trabajo, Ambiente y Salud, 2012).

Como indica Elvira Ramos, profesora de Salud Pública de la Universidad de Murcia, este concepto surgió fruto de las reflexiones del feminismo, en un intento de asegurar la igualdad entre hombres y mujeres en el ámbito familiar y laboral. Si bien se han dado pasos en el camino de la igualdad, todavía estamos lejos de esa meta, por lo que reconocer la Doble Presencia como un riesgo psicosocial más, es un paso importante a la hora de combatirla. Sobre todo si tenemos en cuenta que el $90 \%$ de mujeres que trabajan fuera de casa la sufren, y esta Doble Presencia repercute directamente sobre la salud de dichas mujeres, siendo los síntomas más frecuentes el estrés, la ansiedad, la depresión y los problemas osteomusculares (Ramos, 2008).

Además, la doble presencia es una de las responsables del mantenimiento de la desigualdad laboral entre hombres y mujeres, ya que las obligaciones domésticas impiden a las mujeres dedicarse más intensamente a su puesto de trabajo (algo que si 
pueden hacer los hombres al no tener el peso del trabajo doméstico sobre sus espaldas), de manera que se limita el acceso de éstas a puestos de dirección, el estatus laboral de las mujeres disminuye a medida que aumentan sus obligaciones sociales. (Moreno-Jiménez y Garrosa, 2013).

Es importante mencionar, en relación al riesgo psicosocial que supone para las mujeres la doble presencia, que la situación de crisis económica y el contexto sociodemográfico, agudiza en gran medida este riesgo previamente existente. La razón de ello es por un lado que la reducción de la natalidad y el incremento de la esperanza de vida han provocado un envejecimiento significativo de la población, con el cuidado de personas mayores dependientes que ello implica, un cuidado que tradicionalmente ha recaído sobre las mujeres, más si tenemos en cuenta los últimos recortes en materia de dependencia. Los recortes en los Servicios Públicos (sanidad, educación...) también contribuyen a reforzar la doble presencia por dos motivos fundamentalmente: son los que en mayor medida emplean mujeres, por lo que la destrucción y/o precarización de puestos de trabajo devuelven en gran medida a las mujeres a los hogares; pero también porque son las que más frecuentemente hacen uso de ellos, por ser quienes ejercen tradicionalmente el cuidado de personas dependientes (niños/as, ancianos/as y/o familiares y amigos/as enfermos/as).

No obstante, es importante tener en cuenta, que aunque la opresión de género es la base sobre la que se sustenta esta desigualdad, y que por tanto las mujeres la sufren en mayor medida que los hombres, hay otro componente que modula el efecto de este riesgo psicosocial sobre las mujeres: la clase social o posición socioeconómica de la familia. En algunos casos las mujeres se ven "liberadas" de las obligaciones domésticas si cuentan con apoyo a la hora de realizar dichas "obligaciones". Esta ayuda puede proceder de la contratación de alguna personas que limpie la casa, atienda a los/as niños/as o familiares dependientes; o por otro lado de un reparto equitativo de las tareas de la casa entre los/as componentes de la unidad familiar (y fundamentalmente entre los miembros de la pareja). En el primer caso, no puede pasar desapercibido que el problema de la doble presencia sobre las mujeres no ha desaparecido sino que se ha trasladado a otras mujeres de clase social inferior (y fundamentalmente inmigrantes) que son quienes asumen estos trabajos en casas ajenas. Por lo que podemos afirmar que la doble presencia es una losa muy pesada que sigue recayendo en las mujeres en la actualidad, y que especialmente afecta a las mujeres de clase trabajadora, quienes no pueden deshacerse fácilmente de ella.

Por todo ellos, el objetivo de nuestro trabajo es constatar que la doble presencia es un riesgo psicosocial que afecta casi exclusivamente a las mujeres, así como observar los niveles que los/as trabajadores/as de la Universidad de Granada manifiestan en esta dimensión del ISTAS21, además de analizar cuáles son las propuestas de abordaje para este riesgo psicosocial. El interés por analizar los riesgos psicosociales asociados al contexto laboral del personal docente e investigador (PDI) y del personal de administración y servicios (PAS) de la Universidad de Granada es doble: por un lado el interés por conocer los riesgos psicosociales de los/as trabajadores de nuestro centro de estudio; y por otro analizar las diferencias que podemos encontrar en relación a estos riesgos entre dos poblaciones que a pesar de formar parte de la misma institución ocupan puestos de trabajo muy diferentes (en cuanto a tipo de trabajo realizado, estatus profesional, salario...). A pesar de que la investigación tuvo en cuenta mucho otros riesgos psicosociales, nosotros/as nos centraremos únicamente en la dimensión que hace referencia a la doble presencia, en un intento por analizar profundamente este riesgo psicosocial que evidencia seriamente la existencia de desigualdades entre hombres y mujeres. 
En, concreto las hipótesis que planteamos para el trabajo que nos ocupa son: 1) Las mujeres sufren la doble presencia en mayor medida que los hombres 2) La doble presencia afectará en mayor medida a las mujeres del PAS que a las del PDI.

\section{MÉTODO}

\section{Participantes}

En nuestro estudio hemos contado con una muestra de 105 participantes de los cuales 45 eran hombres y 60 mujeres, todos ellos trabajadores/as de la UGR (tanto PDI y PAS) mayores de 25 años pertenecientes a las facultades de Ciencias de la Educación, Psicología, Filosofía y Letras, Ciencias, Traducción e Interpretación, Económicas y Empresariales, Trabajo Social y Ciencias del Trabajo. 65 de los sujetos pertenecen al grupo profesional del PDI, 33 pertenecientes al PAS y 7 pertenecientes a la categoría "Otros Grupos".

\section{Instrumentos}

COPSOQ. El material que hemos utilizado para llevar a cabo el estudio ha sido una adaptación al castellano del Cuestionario Psicosocial de Copenhague (COPSOQ) del Instituto Nacional de Seguridad y Salud Laboral de Dinamarca que se creó con el objetivo de evaluar los riesgos laborales usando un método válido. La adaptación a la lengua castellana recibió el nombre de ISTAS21 COPSOQ.

EI ISTAS21 cuenta con seis dimensiones psicosociales: Exigencias psicológicas; Trabajo activo y posibilidades de desarrollo; Inseguridad; Apoyo social y calidad de liderazgo; Doble Presencia; Estima.

La que nos interesa para nuestro estudio es la escala de la Doble Presencia, esta escala fue desarrollada en colaboración con el Gabinete de Estudios de Comisiones Obreras (CCOO) de Navarra. La Doble Presencia es una dimensión no comprendida en la escala original, pero muy interesante en cuanto que evidencia en muchos casos, la doble jornada laboral que sufren las mujeres (en casa y fuera de ella), lo cual añade un elemento más a tener en cuenta en el abordaje de la salud laboral. (Moncada, Llorens, Navarro y Krinstensen, 2005)

\section{Procedimiento}

Una vez claros los objetivos de estudio se procedió a pasar el ISTAS 21 en distintas facultades, se pasó a todo tipo de personal con la única condición que fueran trabajadores de la UGR (PAS, PDI y "Otros Grupos"). Antes de comenzar a pasarles el cuestionario se entabló una pequeña conversación en la que se explicaba nuestro objetivo de estudio, además de preguntar edad y antigüedad en el puesto, una vez los participantes terminaron de rellenar los cuestionarios se procedía a realizar una evaluación de sus puntuaciones, cuando obtuvimos los resultados se le preguntaban a los sujetos como creían que podrían mejorar las condiciones adversas en su puesto de trabajo. 


\section{RESULTADOS}

Las puntuaciones obtenidas por los participantes en las diversas dimensiones estudiadas muestran que hay diferencias en las calificaciones, la media obtenida en el trabajo activo fue $2.72(\mathrm{~N}=105)$ con una desviación típica de 0.61 , en apoyo social la media resultante fue $2.68(\mathrm{~N}=105)$ y una desviación típica de 0.67 , en estima la media fue $2.53(\mathrm{~N}=105)$ y una desviación típica de 0.84 , en la dimensión de doble presencia se obtuvo una media de $2.01(\mathrm{~N}=96)$ y una desviación típica 0.85 , en exigencias psicológicas la media fue $1.85(\mathrm{~N}=105)$ y una desviación típica 0.74 y por último en inseguridad se ha obtenido una media de $1.62(\mathrm{~N}=105)$ y una desviación típica 0.95. Cabe mencionar que mientras que las tres primeras dimensiones descritas son positivas, las tres últimas son negativas, lo cual implica que obtener puntuaciones elevadas en trabajo activo, apoyo social y estima, sería síntoma de una buena salud laboral, mientras que puntuar elevado en las tres últimas dimensiones (doble presencia, exigencias psicológica e inseguridad) sería sintoma de mala salud laboral.

Gráfica 1. Riesgos psicosociales en el contexto laboral.

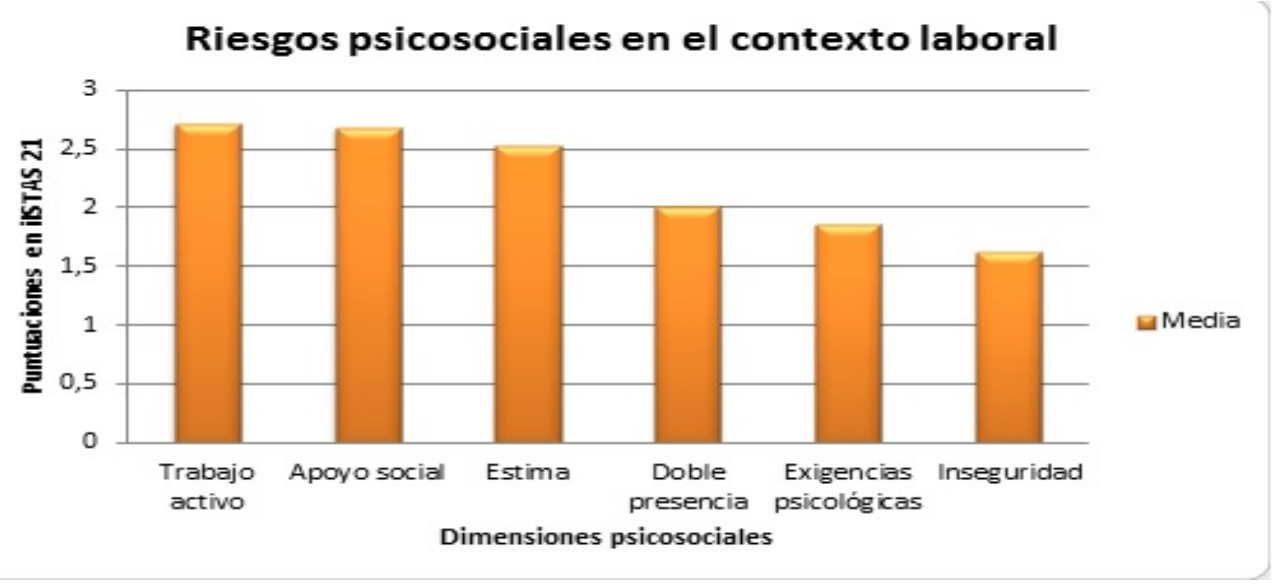

La dimensión escogida en nuestra investigación para analizarla de forma más exhaustiva fue la doble presencia, se aplicó una ANOVA para comparar las diferencias en las puntuaciones respecto al sexo. Lo resultados mostraron $F(1,94)=12$ y $p=0.001$, exponiendo que hay diferencias significativas en esta dimensión respecto al género, teniendo puntuaciones más altas las mujeres $(\mathrm{N}=56$; Media $=2.25)$ que los hombres $(\mathrm{N}=40 ;$ Media=1.68).

Gráfica 2. Influencia de género en las puntuaciones de la doble presencia.

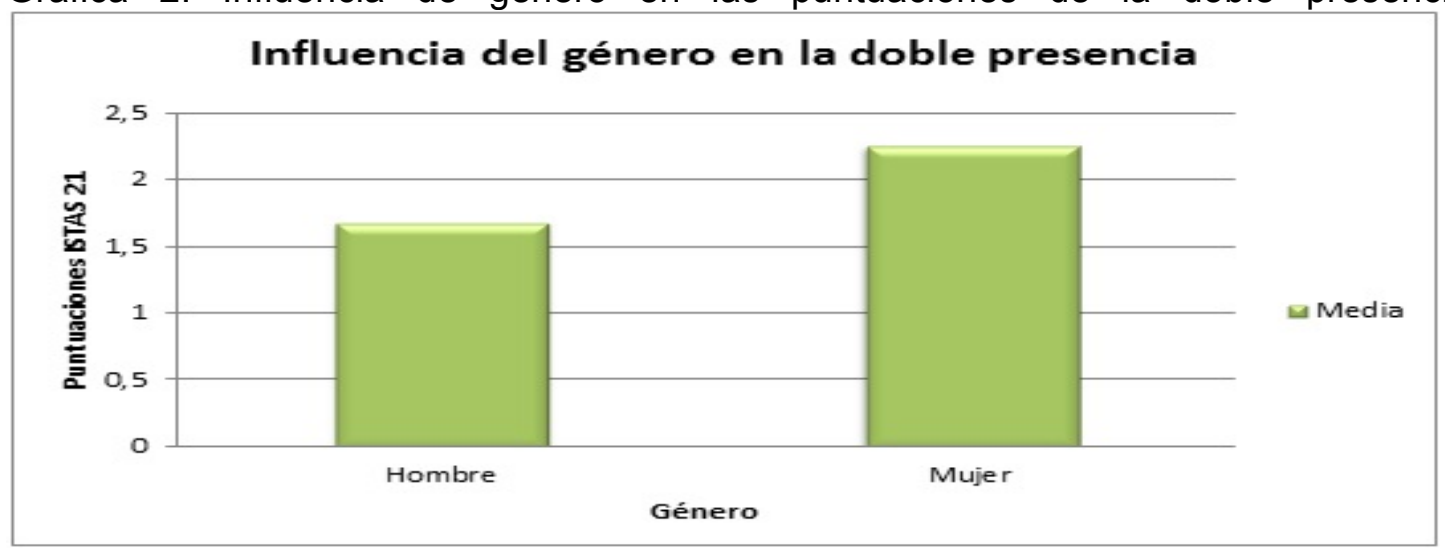


En cuanto al contraste de las puntuaciones obtenidas por los diferentes grupos de trabajadores PDI $(\mathrm{N}=57)$, PAS $(\mathrm{N}=31)$ y otros $(\mathrm{N}=7)$, decir que se realizó una ANOVA obteniendo $F(2,92)=2.91$ y $p=.060$. Mostrando que las diferencias presentadas son parcialmente significativas, puesto que los resultados se encuentran en el límite de la significación, pero pensamos que con una muestra más grande las diferencias podrían ser significativas, puesto que la muestra de ambos grupos fue reducida y no estuvo equilibrada, pudiendo afectar a los resultados presentes.

Gráfica 3. Comparación del personal laboral en las puntuaciones de la doble presencia.

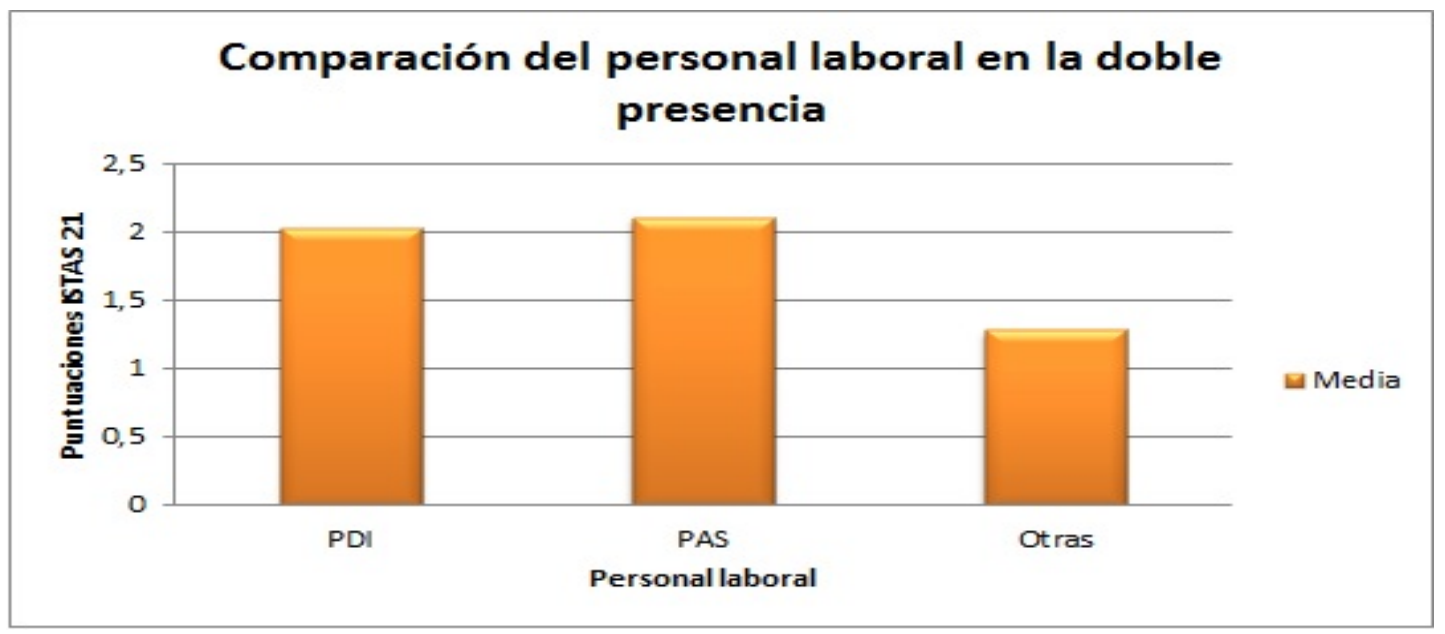

Las propuestas realizadas por los participantes para mejorar la situación de la doble presencia se pueden analizar cualitativamente, las soluciones más recurrentes han sido repartir las tareas domésticas con sus parejas y llevar a cabo una organización más adecuada, las otras propuestas iban dirigidas a que la organización donde trabajan incluyan algún sistema de ayuda con los hijos o pensar menos en las tareas domésticas.

\section{CONCLUSIONES, DISCUSIÓN Y PROPUESTA DE INTERVENCIÓN}

Como se informa, la investigación ha aportado hasta hoy una amplia evidencia del efecto sobre la salud y el bienestar de los riesgos psicosociales. Estamos hablando del bajo

control sobre el contenido y las condiciones de trabajo, las altas exigencias psicológicas,

el bajo apoyo social en la realización del trabajo y de la escasez de recompensas obtenidas a cambio del esfuerzo invertido.

Tras el proceso de evaluación de los riesgos psicosociales llevados a cabo en nuestro estudio (profesionales PDI y PAS) y tras analizar los resultados obtenidos, podemos observar la elevada puntuación obtenida en la dimensión de "doble presencia" en las mujeres. Recalcando así su importancia y el papel que juega en la situación laboral actual de los participantes, considerándola como un factor de riesgo psicosocial y creando una posición más desfavorable para la salud.

La necesidad de responder a las demandas laborales por un lado, y al trabajo doméstico-familiar por otro, puede afectar negativamente a la salud. La realización de 
ambas actividades puede aumentar las demandas y horas de trabajo (creando un aumento de las exigencias psicológicas),así mismo, puede crear un conflicto en la organización del tiempo, en multitud de ocasiones por la incompatibilidad horaria, o por el sentimiento de sobrecarga. Tanto el sexo masculino como el femenino son vulnerables a dicha presión, pero es sin duda al sector femenino a quien le afecta de forma más negativa, como hemos podido comprobar en nuestros resultados.

Conscientes de la importancia que tiene en la salud éste y otros riesgos psicosociales, se deberían emplear todos los recursos posibles en localizarlos y posteriormente en diseñar programas para la implementación de medidas preventivas, con el objetivo último de identificar los aspectos que se deben mejorar en la organización del trabajo, modificar los inapropiados, instaurar cambios etc. para crear un estado positivo y de bienestar laboral para el trabajador/a.

En el marco de las relaciones laborales pueden llevarse a cabo una serie de medidas. Por ejemplo: la negociación colectiva y sectorial de la empresa. Cómo delegados y delegadas de prevención debemos participar en la negociación de medidas para incidir en la conciliación de la vida familiar, personal y laboral, así como también en la negociación del tiempo de trabajo y su distribución. Es necesario que el trabajo doméstico se comparta entre el conjunto de personas que conviven en el hogar, así como mejorar la infraestructura y servicios sociales públicos, en lo que se refiere a la atención y cuidado de la infancia y personas dependientes. (Instituto Sindical de Trabajo, Ambiente y Salud, 2012).

Nuestro objetivo se centrará en conseguir disminuir la "doble presencia" de la mujer, y así conseguiremos evitar los riesgos para su salud y aumentar su tiempo libre. Lo que se trata es de informar y sensibilizar a la población en general sobre los problemas que afectan a las trabajadoras, principalmente.

Incidir sobre esta cuestión es una responsabilidad de todos: instituciones, asociaciones empresariales, administración e individuos particulares, para favorecer y posibilitar la conciliación laboral de hombres y mujeres.

En numerosas ocasiones, las mujeres han tenido que trabajar bajo condiciones precarias de temporalidad, salario y trato, provocando, que las mujeres no puedan promocionar y tengan difícil el acceso a puestos de mayor responsabilidad, renunciando a muchas oportunidades a nivel profesional. Para ello es necesario que se le faciliten las condiciones en el ámbito laboral, mediante la elección de turnos o jornada de trabajo, formación, instauración de guarderías en las empresas, etc.. En este sentido, sería imprescindible actuar también desde la negociación, y establecer por convenio las medidas anteriormente expuestas, así como impulsar la participación sindical de las mujeres. También es imprescindible, mentalizar a la sociedad de la necesidad de llevar a la práctica los Planes de lgualdad de Oportunidades entre hombres y mujeres, mediante programas y cursos, además de psicoeducación.

De igual manera, es preciso luchar por la corresponsabilidad dentro de la pareja, haciendo que el colectivo masculino se implique en las tareas domésticas y adecúe su vida laboral y profesional con las responsabilidades familiares, a través de la sensibilización de los agentes sociales, políticos y sociedad en valores de igualdad. Por lo que se podrían crear programas en los que se desarrollen las habilidades y actividades domésticas (cocinar, lavar, planchar, limpiar, cuidado de hijos, manejo de situaciones, habilidades sociales etc.) dentro de las mismas empresas. 
Por último, es necesario educar a las nuevas generaciones en estos valores bajo la responsabilidad común de las tareas familiares y domésticas, cuánto más pequeños mejor, más garantía de efectividad y mantenimiento en la edad adulta. (Secretaría de la Mujer de CCOO de Navarra, 2001).

\section{BIBLIOGRAFÍA}

Instituto Sindical de Trabajo, Ambiente y Salud. (2012). Guía Sindical: Mujeres, trabajo $y$ salud. [Recuperado el 16 de mayo de 2014 de http://www.cantabria.ccoo.es/comunes/recursos/5/pub100386_Guia_Sindical_ Mujeres,_trabajos_y_salud_.pdf]

Moncada. S, Llorens. C, Navarro. A, Krinstensen. T.S (2005). ISTAS21: Versión en lengua castellana del cuestionariopsicosocial de Copenhague (COPSOQ). Archivo Prevención de Riesgos Laborales, 8 (1), 18-29.

Moreno-Jiménez, B., y Garrosa Hernández, E. (2013). Salud laboral. Madrid: Pirámide.

Ramos, E. (2008). Doble Presencia: Salud y Prevención.En Clave Sindical, 5, 16-18.

Secretaría de la Mujer de CCOO de Navarra. (2001). Doble presencia y doble ausencia. [Recuperado el 16 de mayo de 2014 de http://www.navarra.ccoo.es/comunes/recursos/17441/pub52173_Doble_presen cia_y_Doble_ausencia.pdf]

Universidad de Granada. (2011). Plan de igualdad de oportunidades entre mujeres y hombres de la UGR. [Recuperado el 17 de mayo de 2014 de http://secretariageneral.ugr.es/pages/normativa/planigualdad100311] 